\title{
A CERTAIN CLASS OF FUNCTIONS CONNECTED WITH FUCHSIAN GROUPS.
}

BY PROFESSOR ARNOLD EMCH.

(Read before the American Mathematical Society April 24, 1915.)

1. Consider a Fuchsian group $\Gamma$ of linear substitutions

$$
\begin{aligned}
& V_{i} \equiv z_{i}=\frac{\alpha_{i} z+\beta_{i}}{\gamma_{i} z+\delta_{i}} \quad(i=1,2,3, \cdots) \\
& \alpha_{i} \delta_{i}-\beta_{i} \gamma_{i}=1,
\end{aligned}
$$

that transform the unit circle into itself, and for which the unit circle is a natural boundary. The index $i$ for which $z_{i}$ approaches a point of the boundary we denote by $\infty$, so that $\lim \left(z_{i}\right)=e^{i \phi}$, where $\phi$ may have any value from 0 to $2 \pi$. Let $z_{0}=z$ represent identity. Denote by $R_{0}=R$ the fundamental region in which $z$ lies, and by $R_{1}, R_{2}, \cdots$ the regions resulting from $R$ by the substitutions $V_{i}(i=1,2,3, \cdots)$. Let $e_{i}$ be the greatest "elongation" of the boundary of $R_{i}$, i. e., the maximum distance between two points of the boundary of $R_{i}$; then, according to a theorem due to Bricard,* it is possible to circumscribe a circle $C_{i}$ to the region $R_{i}$, such that its radius does not need to be greater than at most $e_{i} / \sqrt{3}$.

For $i \neq \infty$, the area $A_{i}$ of $R_{i}$, being that of a singly connected region bounded by circular arcs, is finite, so that for the ratio of the area of the circle $C_{i}$ to that of the region $R_{i}$ we have

$$
1<\frac{\pi e_{i}{ }^{2}}{3 A_{i}}<M \quad(i=1,2,3, \cdots),
$$

where $M$ is a positive finite quantity $>1$. But it can be shown that this inequality also exists when $\lim (i)=\infty$, or $\lim _{=\infty}\left(z_{i}\right)=e^{i \phi}$. Hence from (2) we get

$$
3 \Sigma A_{i}<\Sigma \pi e_{i}{ }^{2}<3 M \Sigma A_{i},
$$

* "Théorèmes sur les courbes et les surfaces fermées," Nouvelles Annales de Mathématique, 4. ser., vol. 14, pp. 19-25 (January, 1914). 
in which the sums are extended over the whole group $\Gamma$. As $\Sigma A_{i}=\pi$ is a finite quantity we find that the sum of the areas of all circles $C_{i}$, and consequently the sum of the squares of the radii of all these circles is finite.

2. Choose now within $R$ any two points $a$ and $b$ and a variable point $z$, so that the area formed by the euclidean triangle $z_{i} a_{i} b_{i}$ lies entirely within $C_{i}$. Now

hence

$$
\left|z_{i}-a_{i}\right| \leqq e_{i} ;\left|z_{i}-b_{i}\right| \leqq e_{i},
$$

and

$$
\left|z_{i}-a_{i}\right| \cdot\left|z_{i}-b_{i}\right| \leqq e_{i}{ }^{2}
$$

$$
\sum_{i=0}^{\infty}\left|z_{i}-a_{i}\right| \cdot\left|z_{i}-b_{i}\right| \leqq \sum_{i=0}^{\infty} e_{i}^{2} .
$$

But, according to (3), $\sum_{i=0}^{\infty} e_{i}{ }^{2}$ is a finite quantity. The left side of (4) is therefore an absolutely convergent series, for all values of $z$ within $R$. The condition for uniform convergence within the whole domain is evidently also satisfied, so that we can state

THEOREM I. The series

$$
\sum_{i=0}^{\infty}\left(z_{i}-a_{i}\right)\left(z_{i}-b_{i}\right)
$$

extended over a Fuchsian group $\Gamma$, with the unit circle as a natural boundary and $z, a, b$ lying within the fundamental region of $\Gamma$, is a uniformly convergent series, and defines an analytic function within $R$ that vanishes for $z=a$ and $z=b$ and has no infinities within $R$. The result is still valid when $z_{b}=z_{a}$, so that

$$
\sum_{i=0}^{\infty}\left(z_{i}-a_{i}\right)^{2}
$$

also defines such a function which at $z=a$ has a zero of the second order.

3. The theorem may immediately be generalized. Choose for $z$ and $a$ any two points within the unit circle (excluding the boundary). The straight line joining them is cut by a finite number of polygons $R_{i}$ into the segments $l_{1}, l_{2}, l_{3}, \cdots, l_{r}$. Any substitution $V_{\lambda} \equiv\left(\begin{array}{c}\alpha_{\lambda} \beta_{\lambda} \\ \gamma_{\lambda} \delta_{\lambda}\end{array}\right)$ of the group $\Gamma$ transforms the 
straight segment from $z$ to $a$ into an arc of a circle from $z_{\lambda}$ to $a_{\lambda}$ and the segments $l_{i}$ into arcs $l_{i \lambda}$ intercepted by the corresponding polygons arising from the substitution $V_{\lambda}$. Every arc $l_{i \lambda}$ is subtended by a chord $s_{i \lambda}<l_{i \lambda}<e_{i \lambda}$, where $e_{i \lambda}$ denotes the elongation of the polygon (region) $R_{i \lambda}$. From this follows immediately that

and

$$
f_{\lambda}=\left|z_{\lambda}-a_{\lambda}\right|<e_{1 \lambda}+e_{2 \lambda}+\cdots+e_{r \lambda},
$$

$$
\left(f_{\lambda}\right)^{2}<\left(e_{1 \lambda}+e_{2 \lambda}+\cdots+e_{i \lambda}+\cdots+e_{k \lambda}+\cdots+e_{r \lambda}\right)^{2} .
$$

From the inequality

$$
2 e_{i \lambda} e_{k \lambda}<e_{i \lambda}{ }^{2}+e_{k \lambda}^{2}
$$

we derive without difficulty

$$
2 \sum_{\substack{i=1, k=1 \\ i \neq k}}^{r} e_{i \lambda} e_{k \lambda}<(r-1)\left(e_{1 \lambda}^{2}+e_{2 \lambda}^{2}+\cdots+e_{r \lambda}^{2}\right) .
$$

Now

$$
\sum_{\lambda=0}^{\infty}\left(f_{\lambda}\right)^{2}=\sum_{\lambda=0}^{\infty}\left(e_{1 \lambda}{ }^{2}+e_{2 \lambda}{ }^{2}+\cdots+e_{r \lambda}{ }^{2}\right)+2 \sum_{\lambda=0}^{\infty} \sum_{\substack{i=1, k=1 \\ 1 \neq k}}^{r} e_{i \lambda} e_{k \lambda} ;
$$

hence, according to (5),

$$
\sum_{\lambda=0}^{\infty}\left(f_{\lambda}\right)^{2}<r \sum_{\lambda=0}^{\infty}\left(e_{1 \lambda}{ }^{2}+e_{2 \lambda}{ }^{2}+\cdots+e_{r \lambda}{ }^{2}\right) .
$$

But

$$
\sum_{\lambda=0}^{\infty} e_{i \lambda}^{2}=\sum_{\lambda=0}^{\infty} e_{k \lambda}^{2}
$$

so that (6) reduces to

$$
\sum_{\lambda=0}^{\infty}\left(f_{\lambda}\right)^{2}<r^{2} \sum_{\lambda=0}^{\infty} e_{\lambda}^{2} .
$$

The right side of this inequality is a finite quantity, so that the series on the left side is absolutely convergent. Hence

Theorem II. The series

$$
\sum_{\lambda=0}^{\infty}\left(z_{\lambda}-a_{\lambda}\right)^{2}
$$


extended over a Fuchsian group with the unit circle as a natural boundary, where $z$ and $a$ are any two points within the unit circle and not on the boundary, when $a$ is fixed, is an absolutely and uniformly convergent series of $z$ for all points within and not on the boundary, and represents an analytic function in the neighborhood of all such points. It has a zero of the second order for $z=a$, and has the unit circle as a natural boundary.

4. This theorem admits of a further generalization. Choose any three points $z, z^{\prime}, a$ within and not on the unit circle, and write $f_{\lambda}=\left|z_{\lambda}-a_{\lambda}\right|, \quad g_{\lambda}=\left|z_{\lambda}{ }^{\prime}-a_{\lambda}\right|$. Assuming $f \neq 0$ $g \neq 0$, it is possible to find a positive finite number $M$ such that the ratio $g_{\lambda} / f_{\lambda}<M, \lambda=1,2,3, \cdots$, also when $z_{\lambda}$ approaches a point on the unit circle. We have therefore $g_{\lambda}<M f_{\lambda}$, and

and consequently

$$
f_{\lambda} g_{\lambda}<M f_{\lambda}^{2}
$$

$$
\sum_{\lambda=0}^{\infty} f_{\lambda} g_{\lambda}<M \sum_{\lambda=0}^{\infty} f_{\lambda}^{2}
$$

As the right side of this inequality is absolutely convergent, it follows that

$$
\sum_{\lambda=0}^{\infty} f_{\lambda} g_{\lambda}
$$

is an absolutely convergent series, and that consequently

$$
\sum_{\lambda=0}^{\infty}\left(z_{\lambda}-a_{\lambda}\right)\left(z_{\lambda}^{\prime}-a_{\lambda}\right)
$$

is absolutely and uniformly convergent, and, for $a$ and $z^{\prime}$ constant, defines an analytic function of $z$ for all points within and not on the boundary of the unit circle. It vanishes for $z=a$ and has the unit circle as a natural boundary. Nothing is lost in the convergency proof of (9) by assuming $z$ and $z^{\prime}$ fixed and $a$ as variable. Hence putting in (9) $z=a$, $z^{\prime}=b$ and $a=z$ we may state

THEOREM III. The series

$$
\sum_{\lambda=0}^{\infty}\left(z_{\lambda}-a_{\lambda}\right)\left(z_{\lambda}-b_{\lambda}\right),
$$

where $a$ and $b$ are any two points within and not on the unit circle, is absolutely and uniformly convergent and represents an analytic 
function of $z$ within the unit circle, which is a natural boundary of the function. It has $z=a$ and $z=b$ as zeros.

5. Making use of the proposition that for an analytic function $F(z)$ which within a certain region has the character of a rational function, such that for any point $z_{0}$ of this region $F\left(z_{0}\right)$ exists,

$$
\lim _{z=z^{\prime} \doteq z_{0}}\left(\frac{F(z)-F\left(z^{\prime}\right)}{z-z^{\prime}}\right)=F^{\prime}\left(z_{0}\right)
$$

we may extend theorem III to an even more general type of functions. Let $\mathscr{R}(z)$ be a rational function of $z$ which for $z=0$ does not become infinite. Putting $\left(z_{\lambda}-a_{\lambda}\right)\left(z_{\lambda}-b_{\lambda}\right)$ $=u_{\lambda},\left(z_{\lambda}{ }^{\prime}-a_{\lambda}{ }^{\prime}\right)\left(z_{\lambda}{ }^{\prime}-b_{\lambda}{ }^{\prime}\right)=u_{\lambda}{ }^{\prime}$, where $z^{\prime}, a^{\prime}, b^{\prime}$ denote a set like $z, a, b$, then as $z_{\lambda}(\lambda=\infty)$ approaches a definite point $e^{i \phi}$ on the boundary $a_{\lambda}, b_{\lambda}, a_{\lambda}{ }^{\prime}, b_{\lambda}{ }^{\prime}, z_{\lambda}{ }^{\prime}$ will approach the same point, and $u$ and $u^{\prime}$ will approach zero as a limit. Consequently

$$
\lim _{z_{\lambda} \dot{=} e^{i \phi}}\left\{\frac{\mathscr{R}\left(u_{\lambda}\right)-\mathscr{R}\left(u_{\lambda}{ }^{\prime}\right)}{u_{\lambda}-u_{\lambda}{ }^{\prime}}\right\}=\mathscr{R}^{\prime}(0)
$$

is a finite quantity, and as $\Sigma\left(u-u^{\prime}\right)$ is absolutely and uniformly convergent, also $\sum_{\lambda=0}^{\infty}\left\{\mathscr{R}(u)-\mathscr{R}\left(u^{\prime}\right)\right\}$ will be absolutely and uniformly convergent within the unit circle, except for a finite number of values of $u$ and $u^{\prime}$, which are poles of $\mathscr{R}(u)$, and their congruents in the group $\Gamma{ }^{*}$ Hence, with the expressions for $u, u^{\prime}$ and $\mathscr{R}$ defined as above, we may state

Theorem IV. When $a, b, a^{\prime}, b^{\prime}, z^{\prime}$ are fixed, so that no $u_{\lambda}{ }^{\prime}$ is a pole of $\mathscr{R}(z)$, then

$$
\sum_{\lambda=0}^{\infty}\left[\mathscr{R}\left\{\left(z_{\lambda}-a_{\lambda}\right)\left(z_{\lambda}-b_{\lambda}\right)\right\}-\mathscr{R}\left\{\left(z_{\lambda}{ }^{\prime}-a_{\lambda}{ }^{\prime}\right)\left(z_{\lambda}{ }^{\prime}-b_{\lambda}{ }^{\prime}\right)\right\}\right]
$$

extended over the whole Fuchsian group represents an analytic function of $z$, which has the same poles as those of $\mathscr{R}(u)$ and their congruents, and which has the unit circle as a natural boundary.

It appears that in general these functions are not automorphic in the ordinary sense.

UNIVERSITY OF ILLINOIS.

* For a statement of formula (10) and its applications to trigonometric and elliptic functions see Schottky: "Ueber die Funktionenklasse, die der Gleichung $F\left(\frac{\alpha x+\beta}{\gamma x+\delta}\right)=F(x)$ genügt"; Crelle, vol. 143 (1913), pp. 1-24. 